\title{
Hadron Structure from Lattice QCD
}

\author{
Philipp Hägler* \\ Institut für Theoretische Physik T39 \\ Physik-Department der TU München \\ James-Franck-Strasse \\ D-85747 Garching, Germany \\ and \\ Institut für Theoretische Physik \\ Universität Regensburg \\ D-93040 Regensburg, Germany \\ E-mail: haeglerep2h.de
}

Hadron structure calculations in lattice QCD have seen remarkable progress during recent years. We illustrate the achievements that have been made by discussing latest lattice results for a number of important observables related to nucleon form factors and generalized parton distributions. A particular focus is placed on the decomposition of the nucleon spin $1 / 2$ in terms of quark spin and orbital angular momentum contributions. Results and limitations of the necessary chiral extrapolations based on ChPT will be briefly discussed.

Light Cone 2010 - LC2010

June 14-18, 2010

Valencia, Spain

${ }^{*}$ Speaker. 


\section{Introduction}

Complementary to the countless experimental and theoretical studies of hadron structure during the last decades, ab-initio calculations in the framework of lattice QCD represent a highly promising approach from first principles to a wide range of fundamental hadron properties. Detailed accounts of the advances that have been made on the lattice, in particular with respect to the quark and gluon structure of the pion and the nucleon, can be found in [Org06, Häg07, Zan08, Sch08, Häg10, Ren10].

In this contribution, we concentrate on a small number of results from recent dynamical lattice QCD studies. We begin with an overview of lattice results for the anomalous magnetic moment and the Pauli mean square radius, including a brief discussion of the corresponding chiral extrapolations in the framework of heavy baryon chiral perturbation theory (HBChPT). This will be followed by a presentation of moments of generalized parton distributions (GPDs) obtained by the LHP collaboration. The impact of these lattice results on the decomposition of the nucleon spin in terms of spin- and orbital angular momentum contributions is discussed in the final section, where lattice values for the up- and down-quark angular momentum will also be compared to other experimental, phenomenological and model studies. Due to space limitations, we point the interested reader to Ref. [HMNS09] for a first exploratory lattice study of transverse momentum dependent distributions (TMDs) that already provided interesting insights into non-trivial correlations between spinand momentum degrees of freedom for polarized quarks in a polarized nucleon.

\section{Nucleon form factors}

Significant progress has been made during the recent years in dynamical lattice QCD calculations of the Dirac and Pauli nucleon form factors, $F_{1}\left(Q^{2}\right)$ and $F_{2}\left(Q^{2}\right)$, by a number of collaborations [AKNT06, QCDSF07, $\mathrm{B}^{+} 08, \mathrm{~L}^{+} 08, \mathrm{Y}^{+} 09, \mathrm{~S}^{+} 10, \mathrm{~A}^{+} 09$ ], with pion masses as low as $\approx 300 \mathrm{MeV}$. Very recently, QCDSF/UKQCD presented result for $N_{f}=2$ Wilson fermions reaching down to $m_{\pi} \approx 180 \mathrm{MeV}$ [QCDSF/UKQCD10] (albeit with larger statistical uncertainties at the lowest pion mass). Fundamental observables derived from the form factors are the mean square (ms) radii, $\left\langle r^{2}\right\rangle_{1,2} \propto-\left(d F_{1,2}\left(Q^{2}\right) / d Q^{2}\right)_{Q^{2}=0}$, as well as the anomalous magnetic moment, $\kappa=F_{2}(0)$. A collection of results for the latter in the isovector channel is displayed in Figure 1 as a function of the pion mass. It is encouraging to see that the data points obtained for the different lattice actions and for $N_{f}=2$ and $N_{f}=2+1$ flavors are overall in good agreement within the statistical errors. Despite the steady increase towards smaller pion masses, the lattice results are still about $30 \%$ below the experimental value even at pion masses of $\approx 250 \mathrm{MeV}$. In a first attempt to understand this difference, we have fitted the data below $m_{\pi}=300 \mathrm{MeV}$, including the experimental value, using results from HBChPT in the small scale expansion (SSE) approach [HW02, QCDSF05], as illustrated by the shaded band in Fig. 1 . Fixing the axial nucleon- $\Delta$ coupling to $c_{A}=1.5$, and varying the isovector nucleon- $\Delta$ coupling between $c_{V}=-1.5, \ldots,-3.5 \mathrm{GeV}^{-1}$, we find a good description of the data points with a comparatively large value for the isovector anomalous magnetic moment in the chiral limit, $\kappa^{0, u-d} \sim 5.2, \ldots, 5.4$.

Corresponding results for the isovector Pauli mean square radius are shown in Fig. 2. Although in particular the most recent data points from QCDSF/UKQCD and ETMC show an up- 


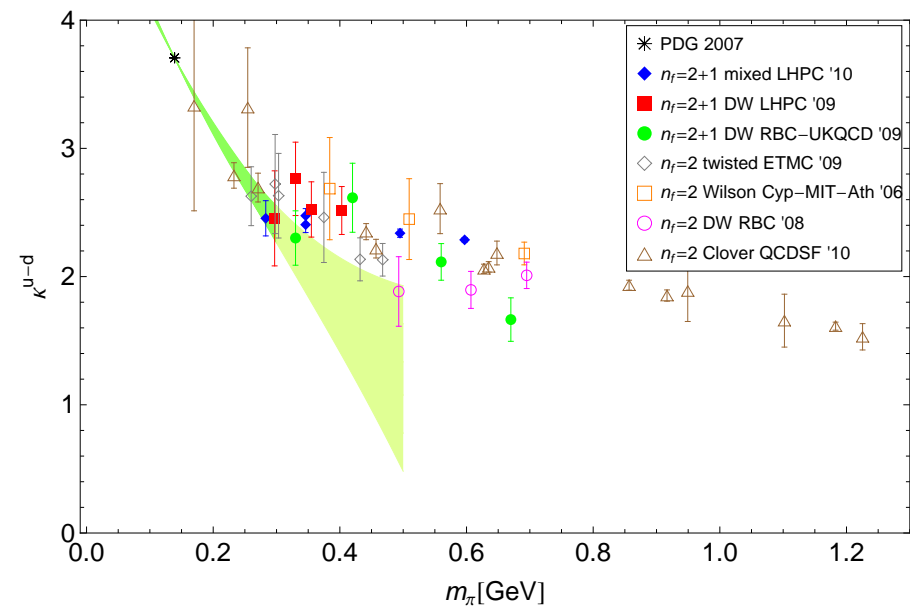

Figure 1: Overview of results for the isovector anomalous magnetic moment obtained by different lattice collaborations. The shaded band is the result of a SSE-HBChPT fit to the data points for $m_{\pi} \leq 300 \mathrm{MeV}$ including the experimental value. References are provided in the text.

ward trend, the lattice values in the range of $m_{\pi} \sim 200 \ldots 300 \mathrm{MeV}$ are remarkable 30-50\% below the phenomenological number of Ref [BHM07]. Qualitatively, this doesn't come as a surprise, since ChPT predicts a linearly diverging isovector Pauli radius in the chiral limit, $\left\langle r^{2}\right\rangle_{2} \propto m_{\pi}^{-1}$ as $m_{\pi} \rightarrow 0$. To see if the results in Fig. 2 can be understood more quantitatively, we have employed the SSE-predictions of Refs. [HW02, QCDSF05] together with the low energy constants obtained from the fit in Fig. 1 to extrapolate the phenomenological value for $\left\langle r^{2}\right\rangle_{2}$ from the physical point to larger pion masses. The immediate change of curvature and strong upwards bending towards larger pion masses, illustrated by the shaded band in Fig. 2, indicates that the SSE HBChPT expansion for $\left\langle r^{2}\right\rangle_{2}$ breaks down at the latest shortly above $m_{\pi}^{\text {phys }}$. This suggest that the range of applicability in $m_{\pi}$ of ChPT for radii is rather limited (at least in this particular approach, to the given order), in particular in comparison to the anomalous magnetic moment discussed above. As before, this is not unexpected since radii are given by derivatives with respect to a small expansion parameter $\left(Q^{2}\right)$ and therefore correspond to a lower order in the chiral expansion relative to, e.g., $\kappa=F_{2}(0)$.

\section{GPDs and the spin structure of the nucleon}

Generalized parton distributions (GPDs) ${ }^{1}$ provide a particularly successful framework for the understanding of hadron structure, that not only encompasses the well known hadron form factors and the PDFs, but that also provides a solid basis for the decomposition of the nucleon spin in terms of spin and (orbital) angular momentum contributions of quarks and gluons. According to [Ji97], the nucleon spin can be given in terms of $x$-moments of the GPDs $H(x, \xi, t)$ and $E(x, \xi, t)$,

$$
\frac{1}{2}=\sum_{q, g} J_{q, g}=\sum_{q, g} \frac{1}{2} \int_{-1}^{1} d x x\left\{H^{q, g}(x, \xi, t)+E^{q, g}(x, \xi, t)\right\}_{t=0}=\sum_{q, g} \frac{1}{2}\left(A_{20}^{q, g}(t)+B_{20}^{q, g}(t)\right)_{t=0},
$$

\footnotetext{
${ }^{1}$ for reviews, we refer to Refs. [GPV01, Die03, BR05]
} 


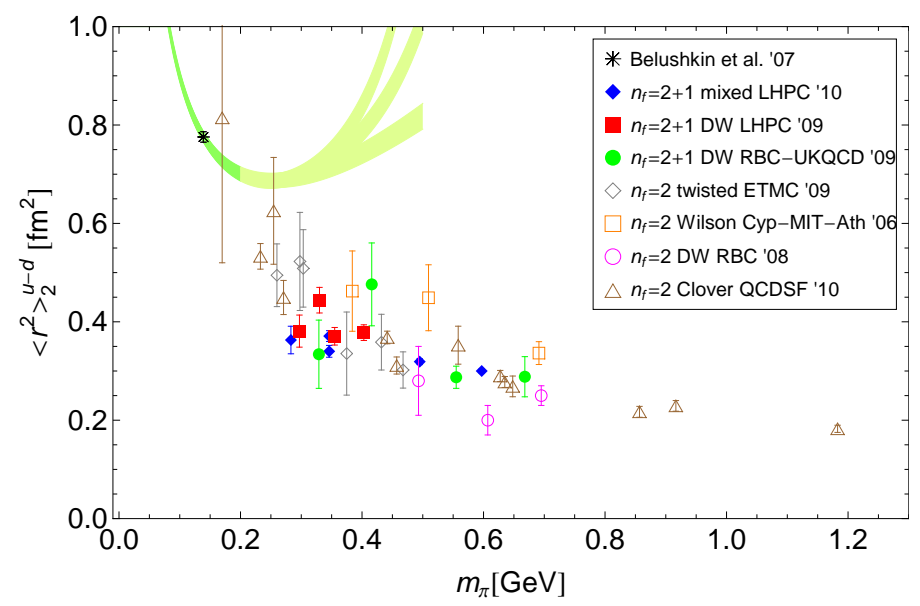

Figure 2: Overview of results for the isovector Pauli mean square radius obtained by different lattice collaborations. The shaded bands are the result of SSE-HBChPT fits to the phenomenological value, using the low energy constants obtained from the fit in Fig. [1]. References are provided in the text.

where $A_{20}$ and $B_{20}$ are generalized form factors (GFFs) that will be discussed in greater detail below. It is important to keep in mind that the total angular momentum of quarks can be further decomposed in a manifestly gauge-invariant manner in terms of quark spin and orbital angular momentum contributions, $J_{q}=\Delta \Sigma_{q} / 2+L_{q}$ [Ji97].

While the GPDs can be measured for example in deeply virtual Compton scattering employing QCD factorization, their extraction from experimental data turns out to be very challenging. This holds in particular for their dependence on $x$ at fixed $\xi$, and therefore also for the $x$-moments in the decomposition in Eq. (3.1). As it turns out, this is different in the framework of lattice QCD, which represents at least in principle a straightforward (but in practice also challenging) approach from first principles to the lowest $x$-moments of PDFs and GPDs.

Significant advances in particular with respect to moments of GPDs have been made since the pioneering calculations by the LHPC and QCDSF collaborations in 2003 [LHPC03, QCDSF04]. A comprehensive lattice study of GPDs by LHPC using a mixed action approach was presented in Ref. [LHPC08]. The discussions in the following sections will be based on a recent update of this study [LHPC10], which includes an additional ensemble at a lower pion mass of $m_{\pi} \approx 300 \mathrm{MeV}$, a factor of 8 increased statistics, and a strongly improved statistical analysis.

The $x$-moments of generalized parton distributions can be written in terms of polynomials in powers of $\xi$ with the GFFs as coefficients. For the $n=2-(x-)$ moments in the unpolarized case, the relevant GFFs $A_{20}, B_{20}, C_{20}$ parametrize the off-forward matrix element of the (symmetric, traceless) energy momentum tensor, e.g. for quarks

$\left\langle P^{\prime}\left|\bar{q} \gamma^{\{\mu} D^{v\}} q\right| P\right\rangle=\left\langle P^{\prime}\left|T_{q}^{\mu v}\right| P\right\rangle=\bar{U}\left(P^{\prime}\right)\left\{\gamma^{\{\mu} \bar{P}^{v\}} A_{20}(t)-\frac{i \Delta_{\rho} \sigma^{\rho\{\mu} \bar{P}^{v\}}}{2 m_{N}} B_{20}(t)+\frac{\Delta^{\mu} \Delta^{v}}{m_{N}} C_{20}(t)\right\} U(P)$,

where $\bar{P}=\left(P^{\prime}-P\right) / 2, \Delta=P^{\prime}-P$, and $t=\Delta^{2}$. Exemplary lattice results from LHPC [LHPC10] for the $t$-dependence of these GFFs in the isovector and isosinglet channels, for a comparatively 

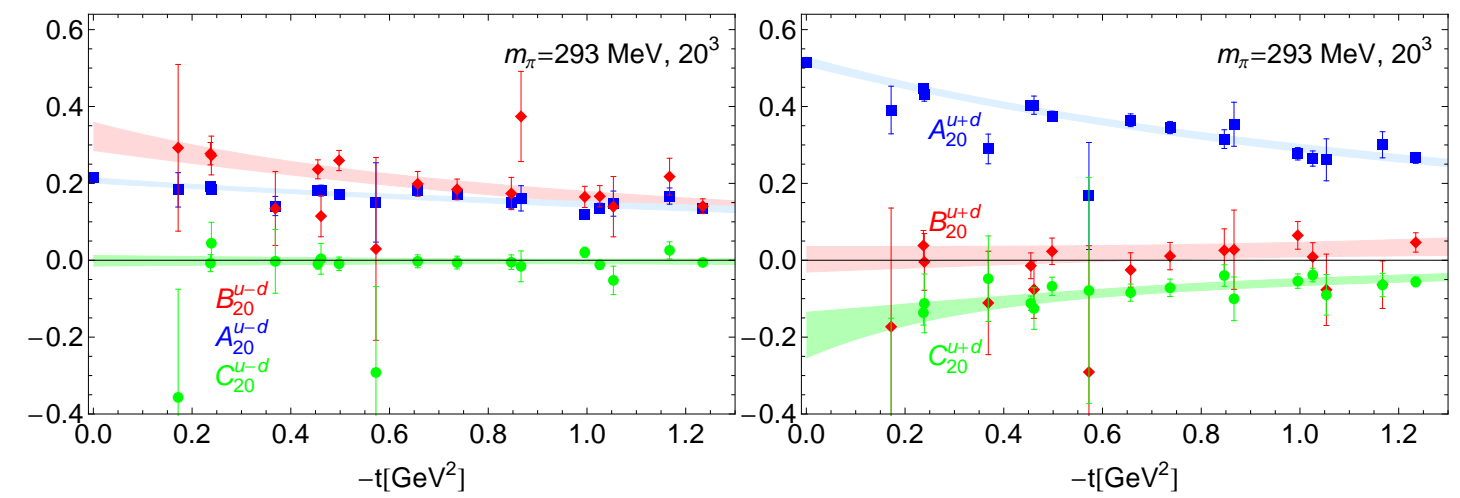

Figure 3: Generalized form factors $A, B, C$ as functions of the squared momentum transfer $t$, in the $\overline{\mathrm{MS}}$ scheme at a scale of $\mu=2 \mathrm{GeV}$, for a pion mass of $m_{\pi} \approx 300 \mathrm{MeV}$ [LHPC10]. The $t$-dependence can be parametrized by, e.g., a dipole ansatz, as illustrated by the shaded error bands.

low pion mass of $\approx 300 \mathrm{MeV}$, are displayed in Fig. 3. We note at least two characteristic features: First, while the $B_{20}$-GFF is dominant in the isovector case, it is very small and fully compatible with zero within the uncertainties for all accessible values of $t$ in the isosinglet channel. Conversely, the GFF $C_{20}$ (which is directly related to the $n=2$-moment of the so-called $D$-term [PW99]) is zero within relatively small errors in the isovector channel, but clearly non-zero and negative for $u+d$ quarks. From the sum of $A_{20}$ and $B_{20}$ at zero momentum transfer, we can compute the quark angular momentum $J_{q}$, cf. Eq. (3.1). The above findings for $B_{20}$ obviously have a significant impact on the decomposition of the nucleon spin in terms of quark and gluon degrees of freedom.

\section{Decomposition of the nucleon spin}

Instead of calculating matrix elements of the quark OAM operator directly on the lattice, we can employ the relation $L_{q}=J_{q}-\Delta \Sigma / 2$, together with corresponding results for the quark spin fraction $\Delta \Sigma$, to compute the OAM carried by the quarks. The numerical results for $u+d$-quarks are shown in Fig. 4 as a function of the squared pion mass. For the extrapolation of $\Delta \Sigma_{u+d}$ to the physical pion mass, we employed the heavy baryon ChPT results of Ref. [DMS07]. Although HBChPT at the given order is most probably not applicable at the accessible pion masses, the two-parameter fit to the lattice data points, indicated by the error band, shows an excellent (but likely accidental) agreement with the value from HERMES [HERMES07], indicated by the cross, at $m_{\pi}^{\text {phys }}$. We used the results of a covariant baryon chiral perturbation theory calculation [DGH08] for the simultaneous extrapolation of the GFFs $A_{20}, B_{20}, C_{20}$ in $m_{\pi}$ and in $t$, in particular to obtain $B_{20}\left(t=0, m_{\pi}^{\text {phys }}\right)$. The results of the fits to the lattice data were subsequently used to compute $L_{q}$, which is shown in Fig. 4 for $q=u+d$ by the diamonds and the error band. Most remarkably, following this procedure, we find a very small OAM contribution of only $L_{u+d} \approx(6 \pm 3) \%$ of $1 / 2$ at the physical pion mass. Note that we obtain a slightly larger value, $L_{u+d} \approx(11 \pm 3) \%$, on the basis of a HBChPT extrapolation of $J_{u+d}$ employing the results of Ref. [CJ02]. These results may be surprising and at first sight even appear to be in clear contradiction to expectations from relativistic quark models, where the quark OAM contributes about $\approx 30-40 \%$ to the total nucleon spin. Moreover, it has been frequently pointed out in the past that non-zero quark orbital angular 


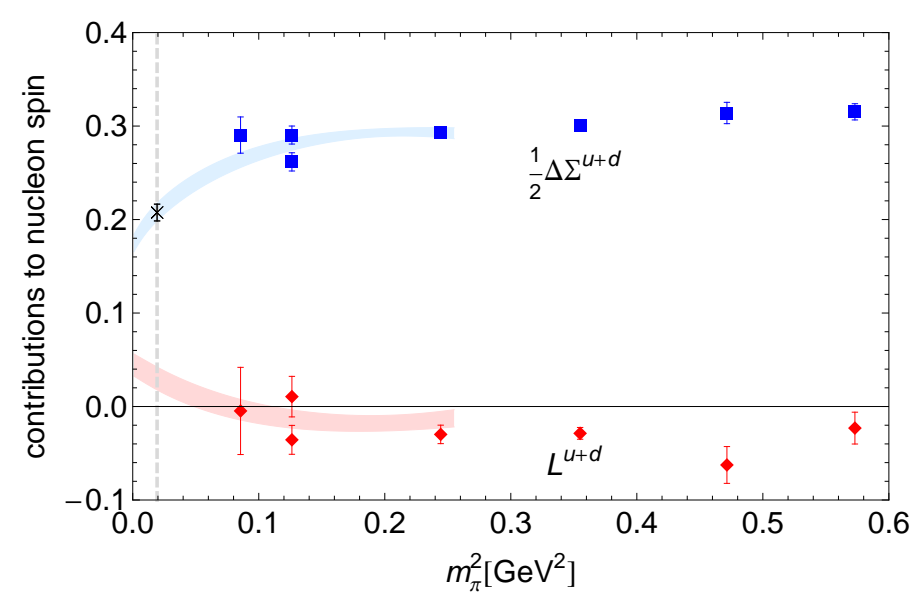

Figure 4: Contributions to the nucleon spin in the $\overline{\mathrm{MS}}$ scheme at a scale of $\mu=2 \mathrm{GeV}$ [LHPC10].

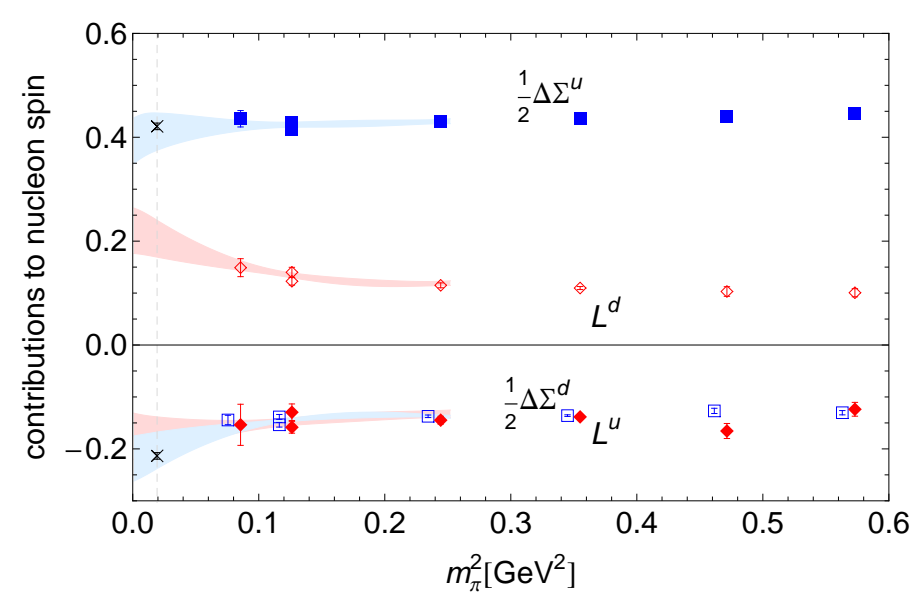

Figure 5: Contributions from up and down quarks to the nucleon spin in the $\overline{\mathrm{MS}}$ scheme at a scale of $\mu=2 \mathrm{GeV}$ [LHPC10].

momentum is strictly required with respect to certain non-vanishing single spin asymmetries related to, e.g., the Sivers effect, as well as for the Pauli form factor $F_{2}\left(Q^{2}\right)$ to be non-zero [BHMS01, BHS02, Bur02, BH04].

Concerning the latter, it is important to be precise in the use of the term quark orbital angular momentum: The lattice results on the one hand correspond to the proton matrix element of the manifestly gauge invariant quark OAM operator as given in, e.g., Ref. [Ji97], which is part of Jis nucleon spin sumrule, see Eq. (3.1) and the adjacent discussion. On the other hand, what is required with respect to a non-vanishing $F_{2}\left(Q^{2}\right)$ and the Sivers effect are light cone wave functions with non-zero orbital angular momentum in an overlap representation of the corresponding matrix elements. Also, the more heuristic approach to explain single spin asymmetries, e.g. the Sivers effect, proposed in Ref. [Bur02], requires a generically non-zero quark orbital motion. The notions of OAM and orbital motion in these contexts are, however, not necessarily in one-to-one correspondence with the gauge invariant $L_{q}$ discussed before.

Furthermore, we note that $L_{q}$ is in general not positive definite. To fully appreciate the potential 


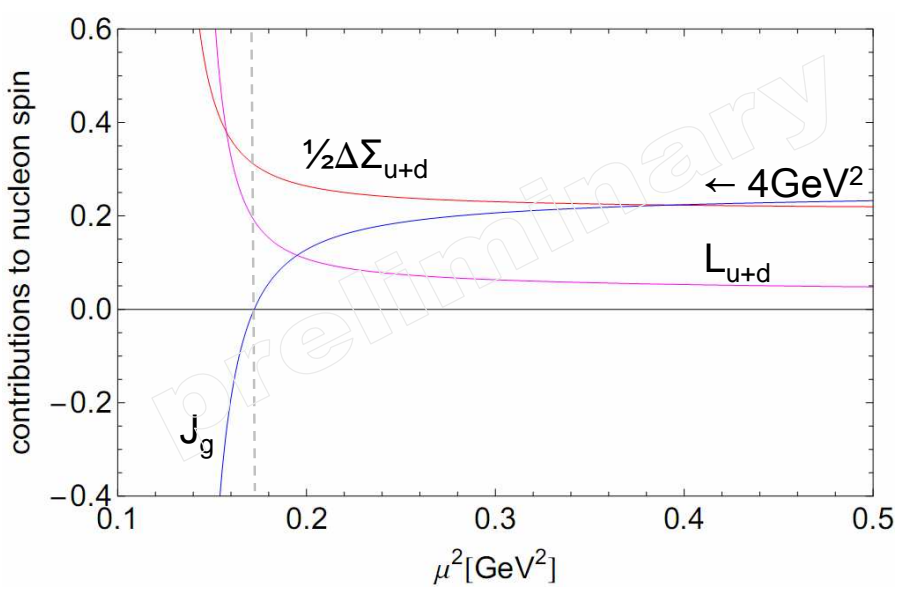

Figure 6: Evolution of contributions to the nucleon spin [AHW10].

consequences of this basic observation, it is useful to study the individual up and down quark OAM contributions to the nucleon spin, which are shown in Fig. 5 as functions of $m_{\pi}^{2}$, together with $\Delta \Sigma_{u, d} / 2$ and the corresponding chiral extrapolations (given by the error bands). It indeed turns out that the orbital angular momentum of up and of down quarks is in each case large and of similar magnitude, but opposite in sign, and therefore nearly cancels in the sum over a wide range of pion masses. From the chiral extrapolations, we find that the individual quark OAM contributions are also substantial at the physical pion mass, amounting to $\left|L_{u, d}\right| \approx 33 \%$. We also note that cancellations of this sort in the isosinglet channel are not unusual. A well known example is the anomalous magnetic moment, which is large both for the proton and for the neutron, $\kappa_{p}=$ $F_{2}^{p}(0) \sim 1.79$ and $\kappa_{n}=F_{2}^{n}(0) \sim-1.91$, respectively. Using isospin symmetry, however, one finds that the sizeable, individual contributions from up and down quarks to $\kappa_{p}$ largely cancel out in the sum, $\kappa_{p}^{u+d} \approx-0.36$. Keeping in mind that $F_{2}\left(Q^{2}=-t\right)$ correspond to the first, and the GFF $B_{20}(t)$ to the second $x$-moment of the GPD $E(x, \xi=0, t)$, we also find this to be perfectly consistent with the cancellation observed above in Fig. 3, where in particular $B_{20}^{u+d}(t) \approx 0$, while $B_{20}^{u-d}(t)$ is large and positive.

In the following, we will briefly address the apparent discrepancy between the common expectations from relativistic quark model calculations that $L_{u+d} \approx 30-40 \%$, and the lattice QCD result of a nearly vanishing total quark $\mathrm{OAM}, L_{u+d} \approx 0$. As has been pointed already out some time ago [WN08, Tho08], the model calculations generically correspond to a low hadronic scale $\ll 1 \mathrm{GeV}$, while a typical renormalization scale for which the lattice results are given is $\mu \sim 2 \mathrm{GeV}$ (e.g. in the $\overline{\mathrm{MS}}$-scheme). Since OAM is a scale and scheme dependent quantity [JTH96], a naive, direct comparison of lattice and model values at the respective scales is therefore in general meaningless. However, one might try to use QCD evolution equations to evolve the lattice values down to the lower model scale, where they may be compared to the model results. To this extent, we show in Fig. 6 the evolution of $\Delta \Sigma_{u+d} / 2$ and $L_{u+d}$ at NLO [AHW10]. Even though the evolution equations are quantitatively not applicable at very low scales, it is still interesting to see that the quark OAM indeed increases substantially at lower $\mu^{2}$. The initial suspicion about a contradiction of lattice and model results, albeit it cannot be ruled out on the basis of these observations, hence appears to be 


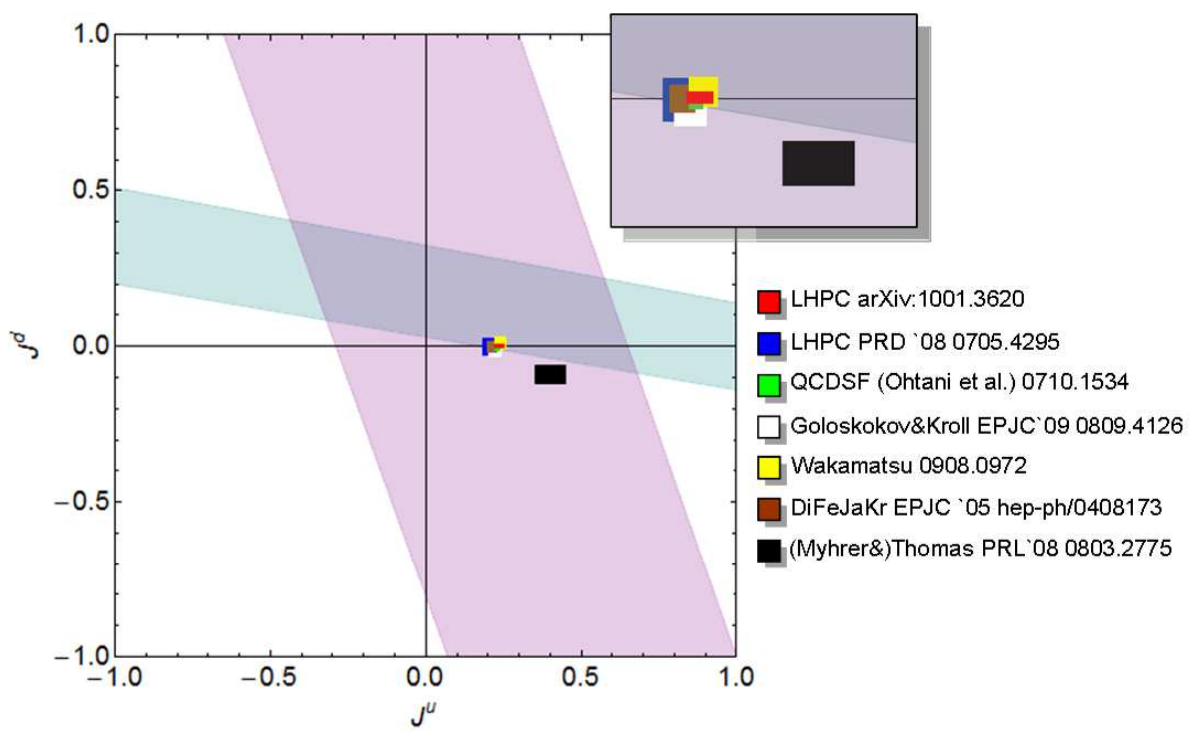

Figure 7: Comparison of quark angular momentum contributions to the nucleon spin, obtained in different lattice (for uncertainties, see footnote ${ }^{2}$ ), model and phenomenological studies $\left[\mathrm{B}^{+} 08\right.$, LHPC08, QCDSF-UKQCD07, Wak10, DFJK05, Tho08], displayed in the $J_{u}, J_{d}$-plane in the style of Refs. [HERMES08, A07], for the $\overline{\mathrm{MS}}$ scheme at a scale of $\mu=2 \mathrm{GeV}$. Note that the shaded bands are mainly given for illustration purposes and only approximately represent the constraints obtained in Refs. [HERMES08, A07], which are still strongly model dependent.

premature.

Finally, we show a comparison of the results for the total quark angular momentum $J_{q}=$ $\left(A_{20}^{q}+B_{20}^{q}\right)_{t=0} / 2$, as obtained from the chiral extrapolations of $A_{20}$ and $B_{20}$ mentioned above, with earlier lattice as well as model- and phenomenological calculations in Fig. 7 in the $J_{u}, J_{d}$-plane. It turns out that the numerical values [LHPC10], $J_{u}=0.236(6)$ and $J_{d}=0.0018(37)$, are in surprisingly good agreement not only with the previous lattice computations, but also with most of the phenomenological and model results, at least when they are evolved to the common renormalization point of $4 \mathrm{GeV}^{2}$. We note in passing that in the lattice calculations, the smallness of $J_{d}$ can be traced back to a cancellation of the separately sizeable spin- and OAM contributions of down quarks, as can be inferred directly from Fig. 5. It will be very interesting to see if the slight discrepancy to the cloudy bag model (CBM) study of Ref. [Tho08] is due to systematic effects, missing contributions or similar on the side of the lattice ${ }^{2}$ and the other approaches, or if it points towards the need for a further improvement of the CBM calculation.

\section{Acknowledgments}

It is a pleasure to thank M. Altenbuchinger, B. Musch, W. Weise and the colleagues from QCDSF/UKQCD and LHPC for successful collaboration. I gratefully acknowledge the support by the DFG Emmy-Noether program, cluster of excellence "Origin and Structure of the Universe", and Sonderforschungsbereich/Transregio 55.

\footnotetext{
${ }^{2}$ most notable issues are: discretization and finite volume effects, missing disconnected contributions, and the so far neglected mixing of quark and gluon operators under renormalization and evolution in the singlet sector
} 


\section{References}

[A07]

$\left[\mathrm{A}^{+} 09\right]$

[AHW10]

[AKNT06]

$\left[\mathrm{B}^{+} 08\right]$

[BH04]

[BHM07]

[BHMS01]

[BHS02]

[BR05]

[Bur02]

[CJ02]

[DFJK05]

[DGH08]

[Die03]

[DMS07]
M. Mazouz et al. (Jefferson Lab Hall A Collaboration), Deeply Virtual Compton Scattering off the neutron, Phys. Rev. Lett. 99, 242501 (2007), 0709.0450.

C. Alexandrou et al., Nucleon form factors with $\mathrm{Nf}=2$ dynamical twisted mass fermions, PoS LAT2009, 145 (2009), 0910.3309.

M. Altenbuchinger, P. Hägler and W. Weise, in preparation, 2010.

C. Alexandrou, G. Koutsou, J. W. Negele and A. Tsapalis, The nucleon electromagnetic form factors from lattice QCD, Phys. Rev. D74, 034508 (2006), hep-lat/0605017.

J. D. Bratt et al., Aspects of Precision Calculations of Nucleon Generalized Form Factors with Domain Wall Fermions on an Asqtad Sea, PoS LATTICE2008, 141 (2008), 0810.1933.

M. Burkardt and D. S. Hwang, Sivers asymmetry and generalized parton distributions in impact parameter space, Phys. Rev. D69, 074032 (2004), hep-ph/0309072.

M. A. Belushkin, H. W. Hammer and U. G. Meissner, Dispersion analysis of the nucleon form factors including meson continua, Phys. Rev. C75, 035202 (2007), hep-ph/0608337.

S. J. Brodsky, D. S. Hwang, B.-Q. Ma and I. Schmidt, Light-cone representation of the spin and orbital angular momentum of relativistic composite systems, Nucl. Phys. B593, 311-335 (2001), hep-th/0003082.

S. J. Brodsky, D. S. Hwang and I. Schmidt, Final-state interactions and single-spin asymmetries in semi-inclusive deep inelastic scattering, Phys. Lett. B530, 99-107 (2002), hep-ph/0201296.

A. V. Belitsky and A. V. Radyushkin, Unraveling hadron structure with generalized parton distributions, Phys. Rept. 418, 1-387 (2005), hep-ph/0504030.

M. Burkardt, Impact parameter dependent parton distributions and transverse single spin asymmetries, Phys. Rev. D66, 114005 (2002), hep-ph/0209179.

J.-W. Chen and X.-D. Ji, Leading chiral contributions to the spin structure of the proton, Phys. Rev. Lett. 88, 052003 (2002), hep-ph/0111048.

M. Diehl, T. Feldmann, R. Jakob and P. Kroll, Generalized parton distributions from nucleon form factor data, Eur. Phys. J. C39, 1-39 (2005), hep-ph/0408173.

M. Dorati, T. A. Gail and T. R. Hemmert, Chiral Perturbation Theory and the first moments of the Generalized Parton Distributions in a Nucleon, Nucl. Phys. A798, 96-131 (2008), nucl-th/0703073.

M. Diehl, Generalized parton distributions, Phys. Rept. 388, 41-277 (2003), hep-ph/0307382.

M. Diehl, A. Manashov and A. Schäfer, Generalized parton distributions for the nucleon in chiral perturbation theory, Eur. Phys. J. A31, 335-355 (2007), hep-ph/0611101. 
[GPV01]

[Häg07]

[Häg10]

[HERMES07]

[HERMES08]

[HMNS09]

[HW02]

[Ji97]

[JTH96]

$\left[\mathrm{L}^{+} 08\right]$

[LHPC03]

[LHPC08]

[LHPC10]

[Org06]

[PW99]
K. Goeke, M. V. Polyakov and M. Vanderhaeghen, Hard exclusive reactions and the structure of hadrons, Prog. Part. Nucl. Phys. 47, 401-515 (2001), hep-ph/0106012.

P. Hägler, Progress in hadron structure physics on the lattice, PoS LAT2007, 013 (2007), 0711.0819.

P. Hägler, Hadron structure from lattice quantum chromodynamics, Phys. Rept. 490, 49-175 (2010), 0912.5483.

A. Airapetian et al. (HERMES Collaboration), Precise determination of the spin structure function $\mathrm{g} 1$ of the proton, deuteron, and neutron, Phys. Rev. D75, 012007 (2007).

A. Airapetian et al. (HERMES Collaboration), Measurement of Azimuthal Asymmetries With Respect To Both Beam Charge and Transverse Target Polarization in Exclusive Electroproduction of Real Photons, JHEP 06, 066 (2008), 0802.2499.

P. Hägler, B. U. Musch, J. W. Negele and A. Schäfer, Intrinsic quark transverse momentum in the nucleon from lattice QCD, Europhys. Lett. 88, 61001 (2009), 0908.1283 .

T. R. Hemmert and W. Weise, Chiral magnetism of the nucleon, Eur. Phys. J. A15, 487-504 (2002), hep-lat/0204005.

X.-D. Ji, Gauge invariant decomposition of nucleon spin, Phys. Rev. Lett. 78, 610-613 (1997), hep-ph/9603249.

X.-D. Ji, J. Tang and P. Hoodbhoy, The Spin Structure of the Nucleon in the Asymptotic Limit, Phys. Rev. Lett. 76, 740-743 (1996), hep-ph/9510304.

H.-W. Lin et al., Nucleon structure with two flavors of dynamical domain- wall fermions, Phys. Rev. D78, 014505 (2008), 0802.0863.

P. Hägler et al. (LHPC Collaboration), Moments of nucleon generalized parton distributions in lattice QCD, Phys. Rev. D68, 034505 (2003), hep-lat/0304018.

P. Hägler et al. (LHPC Collaboration), Nucleon Generalized Parton Distributions from Full Lattice QCD, Phys. Rev. D77, 094502 (2008), 0705.4295.

J. D. Bratt et al. (LHPC Collaboration), Nucleon structure from mixed action calculations using $2+1$ flavors of asqtad sea and domain wall valence fermions, (to appear in Phys.Rev. D) (2010).

K. Orginos, Recent lattice QCD results on nucleon structure, PoS LAT2006, 018 (2006).

M. V. Polyakov and C. Weiss, Skewed and double distributions in pion and nucleon, Phys. Rev. D60, 114017 (1999), hep-ph/9902451.

[QCDSF-UKQCD07] D. Brömmel et al. (QCDSF-UKQCD Collaboration), Moments of generalized parton distributions and quark angular momentum of the nucleon, PoS LAT2007, 158 (2007), 0710.1534.

[QCDSF04] lattice QCD, Phys. Rev. Lett. 92, 042002 (2004), hep-ph/0304249. 
[QCDSF05] M. Göckeler et al. (QCDSF Collaboration), Nucleon electromagnetic form factors on the lattice and in chiral effective field theory, Phys. Rev. D71, 034508 (2005), hep-lat/0303019.

[QCDSF07] M. Göckeler et al. (QCDSF Collaboration), Nucleon form factors on the lattice with light dynamical fermions, (2007), 0709.3370.

[QCDSF/UKQCD10] D. Pleiter et al. (QCDSF/UKQCD Collaboration), Nucleon form factors and structure functions from $N f=2$ clover fermions, PoS Lattice 2010, 153 (2010).

[Ren10] D. B. Renner, Status and prospects for the calculation of hadron structure from lattice QCD, (2010), 1002.0925.

$\left[\mathrm{S}^{+} 10\right] \quad$ S. N. Syritsyn et al., Nucleon Electromagnetic Form Factors from Lattice QCD using 2+1 Flavor Domain Wall Fermions on Fine Lattices and Chiral Perturbation Theory, Phys. Rev. D81, 034507 (2010), 0907.4194.

[Sch08] A. Schäfer, Hadron structure from lattice QCD, Nucl. Phys. A805, 230-239 (2008).

[Tho08] A. W. Thomas, Interplay of Spin and Orbital Angular Momentum in the Proton, Phys. Rev. Lett. 101, 102003 (2008), 0803.2775.

[Wak10] M. Wakamatsu, The role of orbital angular momentum in the proton spin, Eur. Phys. J. A44, 297-303 (2010), 0908.0972.

[WN08] M. Wakamatsu and Y. Nakakoji, Phenomenological analysis of the nucleon spin contents and their scale dependence, Phys. Rev. D77, 074011 (2008), 0712.2079.

$\left[\mathrm{Y}^{+} 09\right] \quad \mathrm{T}$. Yamazaki et al., Nucleon form factors with 2+1 flavor dynamical domain-wall fermions, Phys. Rev. D79, 114505 (2009), 0904.2039.

[Zan08] J. M. Zanotti, Investigations of hadron structure on the lattice, PoS LAT2008, 007 (2008), 0812.3845. 